\title{
We are not our genes
}

\author{
J. H. van Krieken ${ }^{1}$
}

Published online: 7 November 2015

(C) Springer-Verlag Berlin Heidelberg 2015

As haematopathologists, we try to understand haematological disease by looking at tissues. We see a snapshot of cells in their tissue environment. We can see whether they are normal or abnormal, whether there are too many or too few cells, and how they are organised and interact. We can localise enzymes and proteins, measure expression levels, determine DNA alterations and by bringing all this knowledge together, create a fairly complete idea about the disease that manifests itself in the tissue. We use that idea to provide the patient and the haematologist with information that can be used to choose the best possible treatment (or no treatment of course).

In this era of genetics, we are increasingly able to completely sequence the DNA of individuals and tumours. Hereby, we can diagnose quickly many different diseases that are caused by changes in genes, like congenital immune deficiencies or bi-allelic Lynch syndrome. From leukaemias and lymphomas, we get information on the gene alterations that drive the tumour, like BRAF mutations or ALK-fusions. Increasingly, it is suggested that whole genome sequencing will replace traditional forms of diagnosis. Indeed, if a child with mental retardation comes for a diagnosis to a clinical geneticist, physical examination is already replaced by DNA analysis, and I was informed that in Hong Kong,

\section{J. H. van Krieken}

Han.vanKrieken@radboudumc.nl

1 Department of Pathology, Radboud University Medical Centre, P.O. Box 9101, 6500 HB Nijmegen, The Netherlands lung cancer is already diagnosed on a blood sample in a patient with a hard-to-reach pulmonary lesion in case an EGFr mutation is found and the patient is treated with an anti-EGFr approach (in Hong Kong, the incidence of EGFr-mutated lung cancer is quite high in contrast to western countries). In my view, however, sequencing is an important diagnostic tool with much potential, but it will never give the complete picture.

Genes act through proteins and proteins are modified not only by genetic mechanisms. The development of proteomics approaches is therefore likely to give even more information, but replacing genomics by proteomics (which will still take quite some time) will also not tell the whole story. Cells and tissues are so complex that we cannot understand what is going on when we only extract the genes and proteins. Spatial orientation, communication between cells and composition of tissues are quite critical. Analysing tissues therefore will remain an extremely cheap and quick method to provide useful information, but not only in the way it is done today. I am convinced that there is much more information to be extracted. Deep learning approaches, by which image analysis gets new sorts of information from a tissue, automated quantification of structures and proteins in their natural habitat has a great potential as well.

Our brains are well trained to recognise patterns in tissues, detect abnormalities and integrate findings. We are less adequate when we try to quantify or detect new types information: we see what we know (although by looking carefully we can learn). I am convinced therefore that haematopathologists are in a perfect position to take the lead. We are already experienced in integrating information from different sources to provide useful information. By encompassing the new possibilities from 
genetics and computer-assisted pathology, we will enrich our diagnosis.

But finally, we need to understand that a disease in a tissue is only a part of the picture: it is the whole human being in which the process takes place. Physiology, pathology and psychology all together determine the pathogenesis, manifestation and outcome. Good patient management therefore requires another level of integration, the domain of the haematologist. It is therefore so critical that haematopathologists and haematologists communicate intensively. Although communication skills are probably also largely determined by our genes, we know very well that it can be learned: indeed, we are not only our genes. 